\title{
BMJ Open Effect of computerised cognitive training on cognitive outcomes in mild cognitive impairment: a systematic review and meta-analysis
}

\author{
Haifeng Zhang, ${ }^{01,2}$ Jonathan Huntley, ${ }^{1}$ Rohan Bhome, ${ }^{1}$ Benjamin Holmes, ${ }^{1}$ \\ Jack Cahill, ${ }^{3}$ Rebecca L Gould, ${ }^{1}$ Huali Wang, ${ }^{2}$ Xin Yu, ${ }^{2}$ Robert Howard ${ }^{1}$
}

To cite: Zhang H, Huntley J, Bhome $\mathrm{R}$, et al. Effect of computerised cognitive training on cognitive outcomes in mild cognitive impairment: a systematic review and meta-analysis. BMJ Open 2019;9:e027062. doi:10.1136/ bmjopen-2018-027062

- Prepublication history and additional material for this paper are available online. To view please visit the journal (http:// dx.doi.org/10.1136/bmjopen2018-027062).

Received 5 0ctober 2018 Revised 3 April 2019

Accepted 6 June 2019

Check for updates

(c) Author(s) (or their employer(s)) 2019. Re-use permitted under CC BY-NC. No commercial re-use. See rights and permissions. Published by BMJ.

${ }^{1}$ Division of Psychiatry, University College London, London, UK

${ }^{2}$ Peking University Sixth Hospital, Peking University Institute of Mental Health, NHC Key Laboratory of Mental Health (Peking University), National Clinical Research Center for Mental Disorders (Peking University Sixth Hospital), Beijing Dementia Key Lab, Beijing,

China

${ }^{3}$ Institute of Psychiatry,

Psychology and Neuroscience, King's College London, London, UK

Correspondence to Dr Jonathan Huntley; j.huntley@ucl.ac.uk

\section{ABSTRACT}

Objectives To determine the effect of computerised cognitive training (CCT) on improving cognitive function for older adults with mild cognitive impairment (MCl).

Design Systematic review and meta-analysis.

Data sources PubMed, Embase, Web of Science and the Cochrane Library were searched through January 2018.

Eligibility criteria Randomised controlled trials comparing CCT with control conditions in those with $\mathrm{MCl}$ aged 55+ were included.

Data extraction and synthesis Two independent reviewers extracted data and assessed the risk of bias. Effect sizes (Hedges' $g$ and 95\% Cls) were calculated and random-effects meta-analyses were performed where three or more studies investigated a comparable intervention and outcome. Heterogeneity was quantified using the $\mathrm{I}^{2}$ statistic.

Results 18 studies met the inclusion criteria and were included in the analyses, involving 690 participants. Metaanalysis revealed small to moderate positive treatment effects compared with control interventions in four domains as follows: global cognitive function $(g=0.23$, $95 \% \mathrm{Cl} 0.03$ to 0.44$)$, memory $(\mathrm{g}=0.30,95 \% \mathrm{Cl} 0.11$ to $0.50)$, working memory $(\mathrm{g}=0.39,95 \% \mathrm{Cl} 0.12$ to 0.66$)$ and executive function $(\mathrm{g}=0.20,95 \% \mathrm{Cl}-0.03$ to 0.43$)$. Statistical significance was reached in all domains apart from executive function.

Conclusions This meta-analysis provides evidence that CCT improves cognitive function in older people with $\mathrm{MCl}$. However, the long-term transfer of these improvements and the potential to reduce dementia prevalence remains unknown. Various methodological issues such as heterogeneity in outcome measures, interventions and $\mathrm{MCl}$ symptoms and lack of intention-to-treat analyses limit the quality of the literature and represent areas for future research.

\section{INTRODUCTION}

There are currently estimated to be over 46 million people worldwide living with dementia. This number is expected to grow to approximately 131.5 million by $2050 .^{1}$ There is, therefore, an urgent need to develop therapeutic treatments that may delay or prevent

\section{Strengths and limitations of this study}

- This is a comprehensive systematic review and meta-analysis evaluating the effects of computerised cognitive training in older adults with mild cognitive impairment (MCl) on cognitive outcomes.

- We excluded studies that did not use strict clinical diagnostic criteria for $\mathrm{MCl}$ to reduce the heterogeneity often found between participants in $\mathrm{MCl}$ studies.

- Data for four main cognitive domains most significantly affected by $\mathrm{MCl}$ and targeted by cognitive interventions were extracted from individual studies (global cognitive function, episodic memory, working memory and executive function) and where appropriate composite measures were calculated for meta-analyses.

- The studies included in the systematic review are generally of moderate quality, however, several methodological issues may limit the interpretation of results.

- A lack of follow-up data makes it impossible to draw conclusions regarding long-term effects or impact on the prevalence of dementia.

dementia in population groups considered 'at risk'. ${ }^{2}$ Interventions that delay the onset of Alzheimer's disease (AD) by an average of 2 years would decrease the worldwide prevalence rate by 22.8 million cases, ${ }^{3}$ which in turn, would ease the huge burden placed on individuals, families and society. For these reasons, evidence-based interventions that reduce the risk of dementia are urgently required.

Mild cognitive impairment (MCI) refers to an intermediate stage between normal age-related cognitive decline and dementia. ${ }^{4}$ Although many older adults experience a degree of deterioration in cognitive performance, MCI is described as a greater than the expected cognitive decline for an individual's age and education, but without notable interference in everyday functioning. ${ }^{5}$ Within 
the older adult population, the estimated prevalence rate of MCI ranges from $15 \%$ to $20 \% .^{6}$ Although MCI can present with a variety of symptoms, when memory loss is the predominant symptom, it is termed 'amnestic MCI' and is frequently seen as a prodromal stage of $\mathrm{AD} .{ }^{6}$ When individuals have impairments in domains other than memory, it is classified as non-amnestic single-domain or multiple-domain MCI and these individuals are believed to be more likely to convert to other types of dementia. ${ }^{6}$

The lack of therapeutic benefit or delay in progression from MCI to $\mathrm{AD}$ with pharmacological interventions has meant that the focus has shifted towards non-pharmacological interventions. ${ }^{7}$ Cognitive remediation is the term used for interventions designed to mediate cognitive decline and can be typically identified as involving one of three different approaches: cognitive stimulation (CS), cognitive rehabilitation (CR) and cognitive training (CT). Interventions based on CS and CR are more focused on individuals with established dementia, often with the aim of overcoming specific difficulties with daily living and improving general quality of life. In comparison, CT can be used for subjects without significant cognitive or functional difficulties, and is therefore well suited for individuals with MCI.

CT refers to interventions that aim to improve cognitive domains through repeated practice on theoretically driven skills and strategies. ${ }^{8}$ Each CT exercise aims to target one or two specific domains in an adaptive manner with a possibility of transfer effects whereby performance in other untrained cognitive domains is also improved..$^{9}$

Computerised CT (CCT) uses computers for the delivery of the intervention and differs from traditional CT, which usually incorporates face-to-face contact with a professional and paper-and-pencil paradigms. ${ }^{8}$ CCT has several advantages including cost-effectiveness, increased accessibility and ability to customise the content and difficulty of the training. ${ }^{10-12}$ Research involving older adults has found that CCT programmes are associated with high satisfaction levels, and that they are also a feasible option for individuals with MCI, with equal or better adherence rates when compared with traditional CT. ${ }^{10}{ }^{13}$ In addition, evidence suggests that studies using CCT show a pattern of stronger effect sizes and enhanced generalisation of benefits compared with traditional strategy training in MCI. ${ }^{14}$ A previous meta-analysis found that CT is not effective in people with established dementia. ${ }^{15}$ However, there is growing interest as to whether CCT has the potential to prevent or slow the progression from MCI to dementia particularly given the association between higher participation in mental activity and reduced dementia risk. ${ }^{16}$

Studies investigating the effectiveness of CT in improving cognitive performance in people with MCI have demonstrated small to moderate improvement but existing research suffers from methodological concerns and limitations. ${ }^{14-17-19}$ CT research in individuals with MCI has been criticised for the failure to include an appropriate control group, ${ }^{20-22}$ use of subsets of participants from previous studies ${ }^{23}$ and pooling of MCI data with that from non-impaired adults ${ }^{24}$ as well as those with probable $\mathrm{AD} .{ }^{25-27}$ Another issue raised in treatment studies has been the use of ecologically valid outcome measures. For example, the inclusion of functional outcome measures is important to monitor progression from MCI to dementia but given that individuals with MCI are, by definition, not significantly impaired in functioning, it is a challenge to measure the functional effects of the intervention. ${ }^{17}$

CCT is far from a single construct and factors such as the content, platform, context and dose of training may differ. ${ }^{28}$ Unfortunately, despite increasing scientific scrutiny, there is a limited understanding as to which, if any, dimensions are associated with cognitive benefit. Ideally, critical analysis of research using CCT for MCI would reveal insight into which specific components of CCT are necessary for it to be effective, however, it is important to establish the overall effect of CCT on individuals with MCI.

Systematic reviews and meta-analyses of cognitive interventions in MCI have reported mixed results, ${ }^{1929-34}$ and when exploring the effect of cognitive training in MCI have largely not distinguished between studies evaluating computerised and non-computerised training. This makes it difficult to draw conclusions, specifically on the efficacy of CCT in MCI. For example, a systematic review by Ge et al summarised the findings of CCT studies among people with MCI, however, no meta-analyses were performed and the review included non-randomised controlled studies, studies that combined CCT with other interventions and studies not using Petersen's core MCI diagnosis criteria, making it challenging to draw rigorous conclusions. ${ }^{35}$ A previous meta-analysis by Hill et al specially explored the effectiveness of CCT in MCI on cognition and behavioural outcomes, ${ }^{32}$ however, the field is progressing rapidly, as highlighted by Ge $e t a l$ s observation that $42 \%$ of the studies in their review were published between 2016 and $2017,{ }^{35}$ and further relevant studies have been published subsequently. ${ }^{32}{ }^{36-38}$ Another more recently published meta-analysis by Gates $e t$ al only included studies where the intervention period lasted for more than 12 weeks and excluded a significant number of studies with shorter training duration. ${ }^{39}$ Thus, it is necessary to conduct an updated meta-analysis to include more recent articles and all intervention durations.

This paper investigates the effect of CCT on improving cognitive outcomes in individuals diagnosed with MCI using random-effects meta-analyses. To address some of the problems identified in the literature, only peer-reviewed randomised controlled trials (RCTs) were selected and cognitive outcome measures were extracted for analysis. Variables that may moderate the effect of CCT, such as the type of programme or dose of the intervention, were reviewed. The purpose of the current review was to: (1) evaluate the effect of CCT in older adults with MCI on cognitive outcomes; (2) evaluate the content and methodological quality of the intervention studies and (3) suggest future directions in CCT research in this group based on findings. 


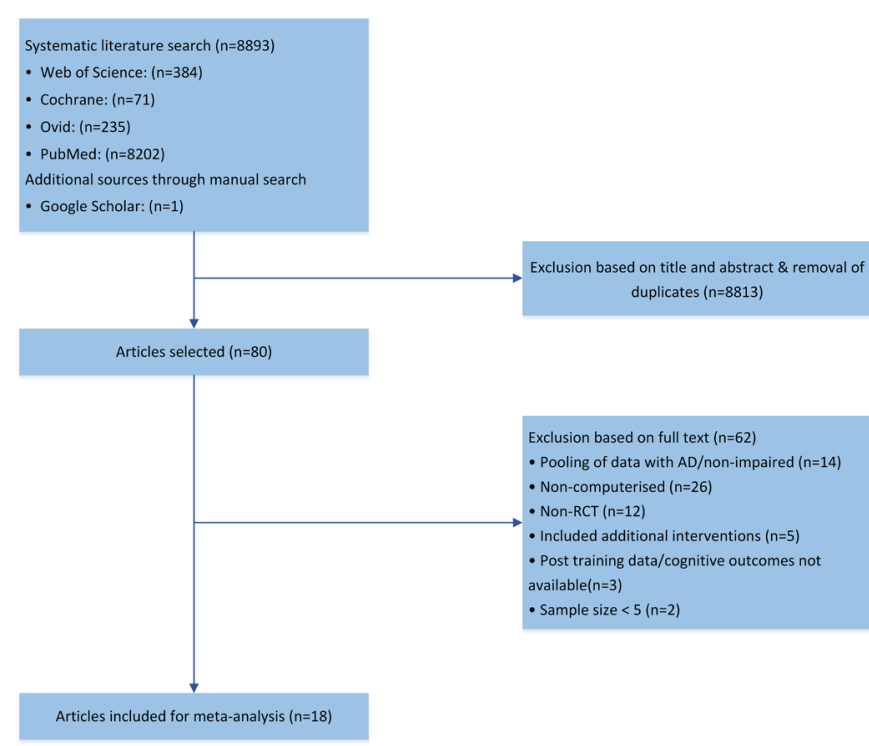

Figure 1 Flow chart of the study selection process. AD, Alzheimer's disease; RCT, randomised controlled trial.

\section{MATERIALS AND METHODS}

\section{Search strategy and selection criteria}

A literature search was completed during January 2018 of four online literature databases and trial registers: PubMed, Embase, Web of Science and Cochrane Library. The search terms are shown in online supplementary table 1. Previous meta-analyses and systematic reviews of cognitive interventions in MCI were also searched. Furthermore, reference lists of included studies were manually scanned for additional relevant papers.

\section{Inclusion and exclusion criteria}

Types of studies: Published, peer-reviewed studies with an RCT design investigating the use of CCT interventions in older people with MCI were considered for inclusion. Studies were included if sufficient data were available for calculation of effect sizes in each treatment arm (unavailable information was requested from authors and included if obtained). The date of publication was not limited, but only studies published in English were included.

Participants: Inclusion criteria were a mean age of participants greater than 55 years, a diagnosis of MCI using core criteria according to Petersen ${ }^{4}$ and no other psychiatric diagnosis or neurological disorder. The number of participants in each arm needed to be at least five. Studies with non-impaired older people or those with probable $\mathrm{AD}$ were excluded unless separate data for participants with MCI were provided.

Types of interventions: Studies were included if they compared any CCT intervention, administered on a personal computer (PC) or gaming console, to an active or non-active control. Computerised training had to represent the primary intervention, not simply one of multiple broader non-computerised cognitive interventions, in order to be included. Active controls were classified as interventions that controlled for non-specific therapeutic effects, whereas non-active control groups included waiting list conditions, treatment as usual or a non-matched minimal intervention. Each study was independently screened, selected for inclusion and its data extracted by independent researchers. Any disagreements were resolved through discussion with another author.

Types of outcome measures: We focused on cognitive domains that are reported to be most significantly affected by MCI and targeted by cognitive interventions, namely episodic memory, executive function, working memory/ attention and global cognitive function. ${ }^{40}$ Available data from all relevant cognitive outcomes were extracted. Cognitive outcomes used in the included studies and their classification into the main cognitive domains are shown in online supplementary table 2.

\section{Risk of bias assessment}

The Cochrane Collaboration Risk of Bias tool was used to assess study methodological quality. ${ }^{41}$ Risk of bias was assessed in multiple domains: sequence generation, allocation concealment, blinding of participants and investigators, incomplete outcome data and selective reporting of outcomes. In each of these categories, the methodological quality of each assessed domain was rated as 'low risk', 'unclear' or 'high risk'. Studies were excluded if unsure or high risk in all assessed domains.

\section{Statistical analysis}

Intervention and control groups' postintervention outcome scores were compared using Review Manager software V.5.3. The program uses Hedges' adjusted $\mathrm{g}^{42}$ to calculate a standardised mean difference which is adjusted for small sample bias. Pooling of standardised mean Hedges' g estimates of $<0.30, \geq 0.30$ and $<0.60$ and $\geq 0.60$ were considered small, moderate and large, respectively. Meta-analyses were performed where three or more studies investigated a comparable intervention and outcome using a random-effects model. Heterogeneity was quantified using the $\mathrm{I}^{2}$ statistic, considered as low, moderate or large when at $25 \%, 50 \%$ or $75 \%$, respectively. ${ }^{43}$ Where a study reported multiple outcome measures for one cognitive domain (eg, within memory function), a composite measure was calculated to provide a single quantitative measure for meta-analysis. ${ }^{44}$ Publication bias was examined using funnel plots. We also performed subgroup analysis and meta-regression using the 'metafor' program in R (https:/ / www.R-project.org/), for example, we compared the effectiveness of single and multidomain training. Furthermore, we subgrouped studies with a training dose of less than 10 hours and more than 30 hours to see if there is a dose-response correlation. We also compared studies with active versus non-active control conditions, following a reviewer's suggestion. Sensitivity analyses were performed to identify potential sources of heterogeneity. Further details of statistical methods are found in the online supplementary material (see online supplementary appendix 1). 
Table 1 Characteristics of studies using computerised cognitive training in persons with $\mathrm{MCl}$

\begin{tabular}{|c|c|c|c|c|}
\hline Author and year & CCT group $N$, age, education & Control group $\mathrm{N}$, age, education & CCT type & $\begin{array}{l}\text { Total } \\
\text { hours }\end{array}$ \\
\hline Barban et al, $2016^{67}$ & $\mathrm{n}=46$, age $=74.4(5.7)$, edu $=9(4.3)$ & $\mathrm{n}=60$, age $=72.9(6.0), \mathrm{edu}=11(4.7)$ & Multidomain & 24 \\
\hline Ciarmiello et al, $2015^{52}$ & $\mathrm{n}=15$, age $=71.2(7.7)$, edu=9.3 (3.0) & $\mathrm{n}=15$, age $=72.0(7.1)$, edu $=7.8(2.6)$ & Multidomain & 24 \\
\hline Djabelkhjr et al, $2017^{36}$ & $\begin{array}{l}n=10, \text { age }=75.2(6.4), \text { edu }=60.0 \% \text { of } \\
\text { college level }\end{array}$ & $\begin{array}{l}n=10, \text { age }=78.2(7.0), \text { edu }=44.4 \% \text { of } \\
\text { college level }\end{array}$ & Multidomain & 18 \\
\hline Fiatarone, et al $2014^{50}$ & $n=24$, age $\geq 55$, edu $=n / s$ & $\mathrm{n}=27$, age $\geq 55$, edu $=\mathrm{n} / \mathrm{s}$ & Multidomain & 80 \\
\hline Finn and McDonald, $2015^{69}$ & $\mathrm{n}=12$, age $=72.8(5.7)$, edu=13.8 (3.0) & $\mathrm{n}=12$, age $=75.1(7.5)$, edu $=13.7(2.8)$ & Memory & $n / s$ \\
\hline Gagnon and Belleville, $2012^{51}$ & $n=12$, age $=67.0(7.8)$, edu=15.0 (4.6) & $n=12$, age $=68.4(6.0)$, edu=13.1 (5.7) & $\begin{array}{l}\text { Attentional } \\
\text { control }\end{array}$ & 6 \\
\hline Gooding et al, 2016 study $1^{70}$ & $\mathrm{n}=31$, age $=75.6$ (8.8), edu=15.1 (2.6) & $\mathrm{n}=10$, age $=75.6(8.8)$, edu=15.1 (2.6) & Multidomain & 30 \\
\hline Gooding et al, 2016 study $2^{70}$ & $\mathrm{n}=23$, age $=75.6(8.8)$, edu=15.1 (2.6) & $\mathrm{n}=10$, age $=75.6(8.8)$, edu=15.1 (2.6) & Multidomain & 30 \\
\hline Hughes et al, $2014^{13}$ & $\mathrm{n}=10$, age $=78.5(7.1)$, edu=13.8 (2.4) & $n=10$, age $=76.2(4.3), e d u=13.1(1.9)$ & Multidomain & 36 \\
\hline Hyer, et al $2016^{53}$ & $\begin{array}{l}\mathrm{n}=34, \text { age }=75.1(7.4), \text { edu }=70 \% \\
\text { secondary }\end{array}$ & $\begin{array}{l}\mathrm{n}=34, \text { age }=75.2(7.8), \text { edu }=66 \% \\
\text { secondary }\end{array}$ & $\begin{array}{l}\text { Working } \\
\text { memory }\end{array}$ & 16.7 \\
\hline Lin et al, $2016^{54}$ & $\begin{array}{l}n=10, \text { age }=72.9(8.2), \text { edu }=90.0 \% \text { of } \\
\text { college level }\end{array}$ & $\begin{array}{l}n=11, \text { age }=73.1(9.6), \text { edu }=54.5 \% \text { of } \\
\text { college level }\end{array}$ & $\begin{array}{l}\text { Processing } \\
\text { speed }\end{array}$ & 24 \\
\hline Rosen et al, $2011^{23}$ & $\mathrm{n}=6$, age $=70.7(10.6)$, edu=16.7 (0.8) & $\mathrm{n}=6$, age $=78.0(7.9), \mathrm{edu}=18.3(1.5)$ & $\begin{array}{l}\text { Processing } \\
\text { speed }\end{array}$ & 36 \\
\hline Rozzini et al, $2007^{72}$ & $n=15$, age $=63-78$, edu $=n / s$ & $\mathrm{n}=22$, age $=63-78$, edu $=\mathrm{n} / \mathrm{s}$ & Multidomain & 60 \\
\hline Savulich et al, $2017^{38}$ & $\begin{array}{l}n=21, \text { age }=75.2(7.4), \text { edu=15.9 (1.3) } \\
\text { (age left school) }\end{array}$ & $\begin{array}{l}n=21, \text { age }=76.9(8.3) \\
\text { edu=16.0 (2.1) (age left school) }\end{array}$ & Memory & 8 \\
\hline
\end{tabular}

$\mathrm{CCT}$, computerised cognitive training; $\mathrm{MCl}$, mild cognitive impairment; n/s, not stated.

\section{Patient and public involvement}

There was no direct patient or public involvement in this review.

\section{RESULTS}

\section{Description of studies}

The Preferred Reporting Items for Systematic Reviews and Meta-analyses checklist was used to guide reporting of results. ${ }^{45}$

Following the initial literature review, a total of 8893 studies were found. Of these, 8875 were excluded and 18 studies met inclusion criteria. Figure 1 presents a flow chart of study selection. The total number of participants included was 690 and the brief summary characteristics of each study are presented in table 1 and detailed in online supplementary table 3. Sample sizes ranged from 12 to 106 , and drop-out rates ranged from $0 \%$ to $32 \%$. One study was excluded from the meta-analysis because of suspected inclusion of participants with probable $\mathrm{AD}$ based on the reported average Mini-Mental State Examination (MMSE) score. ${ }^{46}$ Another two studies were excluded from the meta-analysis as postintervention cognitive data could not be obtained. ${ }^{47} 48$

Table 2 Results of meta-analysis of computerised cognitive training (CCT) on cognitive domains

\begin{tabular}{|c|c|c|c|c|c|}
\hline Analysis of CCT & No of studies & $\begin{array}{l}\mathbf{N} \\
\text { Tx*/control }\end{array}$ & $\begin{array}{l}\text { Pooled effect size g } \\
(95 \% \mathrm{Cl})\end{array}$ & $\begin{array}{l}\text { Overall effect: } \\
Z \text { (p value) }\end{array}$ & $\begin{array}{l}\text { Heterogeneity: } I^{2} \% \\
\text { (p value) }\end{array}$ \\
\hline Global cognition & 11 & $258 / 245$ & 0.23 (0.03 to 0.44$)$ & $z=2.22, p=0.03$ & $6 \% p=0.39$ \\
\hline Working memory & 5 & $82 / 83$ & 0.39 (0.12 to 0.66$)$ & $z=2.85, p=0.004$ & $0 \% p=0.81$ \\
\hline Executive function & 11 & $171 / 182$ & $0.20(-0.03$ to 0.43$)$ & $z=1.74, p=0.08$ & $51 \% p=0.03$ \\
\hline
\end{tabular}

${ }^{\star} \mathrm{T} x=$ training group. 
A total of 13 studies reported outcomes assessing memory, 5 studies reported outcomes assessing working memory, 11 studies reported outcomes assessing executive function and 11 studies reported global cognitive functioning outcomes (see table 2).

\section{Quality of studies}

The quality of each study was evaluated in regard to certain methodological aspects and summarised in online supplementary figure 1 . Eleven of the 18 studies did not report blinding of participants.

\section{Participant characteristics}

The total number of participants from all studies included was 690 (CCT: $n=351$, mean group size: $n=20$, control: $n=339$, mean group size: $n=19$ ). The average age of participants in both conditions was 73.4 years. $52.5 \%$ of all participants were male. The disparity and lack of reporting of the ratio of participants' years of education precluded mean calculations, although the available data suggest most participants had at least secondary school education. The pooled average baseline score for the MMSE was 26.9 in both groups, although the range of scores indicated heterogeneity within participants.

\section{Cognitive training interventions}

Interventions were mostly delivered on a PC, using commercially available or purpose built CT packages, with two studies using a video game on a games console. ${ }^{13} 38$ All interventions were specifically designed to improve various aspects of cognition. The most common type of intervention used was multidomain (11/18 studies), where the programme targeted two or more cognitive domains. In the seven single domain intervention studies, three evaluated memory training and executive function training while one used working memory training. The dose and duration of the CT intervention was variable, with the total length of training ranging from $4^{49}$ to 80 hours $^{50}$ and the duration of training from $2^{51}$ to 26 weeks. ${ }^{50}$

\section{Outcome measures}

Online supplementary table 2 summarises the 60 different cognitive outcome measures used by studies included in the meta-analyses. A considerable variability in measures reported was also noted; only three outcome measures were reported three or more times; seven studies used the MMSE as a measure of global cognition, three studies used paired-associates learning to measure memory and in four studies used the trail making test as a measure of executive function.

\section{Meta-analysis of specific outcomes}

Separate meta-analyses were conducted on four different cognitive domains. The most commonly tested domains were memory, with 13 studies exploring this domain. The results of the meta-analyses are presented in table 2.

\section{Global cognition function}

Overall, there was a significant benefit of CCT on global cognition compared with the control group. The meta-analysis revealed a small but statistically significant pooled effect size of $0.23(95 \%$ CI 0.03 to $0.44, \mathrm{z}=2.22$, $\mathrm{p}=0.03)$ with low heterogeneity between studies $\left(\mathrm{I}^{2}=6 \%\right)$ (see figure 2). The funnel plot did not reveal significant asymmetry (see online supplementary figure 2). The effect size across active-controlled trials $(\mathrm{n}=7, \mathrm{~g}=0.23$, $95 \% \mathrm{CI}-0.05$ to $0.51, \mathrm{I}^{2}=27 \%$ ) was smaller than that of trials with non-active control groups $(n=4, g=0.31,95 \%$ CI -0.06 to $0.68, \mathrm{I}^{2}=0 \%$ ) (see online supplementary figures 3 and 4), but was not statistically significantly different $(\mathrm{z}=-0.11, \mathrm{p}=0.91)$.

\section{Memory}

The pooled effect size of CCT on memory outcomes, when compared with control conditions, was moderate and statistically significant $(\mathrm{g}=0.30,95 \%$ CI 0.11 to 0.50 , $\mathrm{z}=3.03, \mathrm{p}=0.002)$, with moderate heterogeneity between studies $\left(\mathrm{I}^{2}=46 \%\right)$ (see figure 3 ). The funnel plot did not reveal significant asymmetry (see online supplementary figure 5). The effect size across active-controlled trials

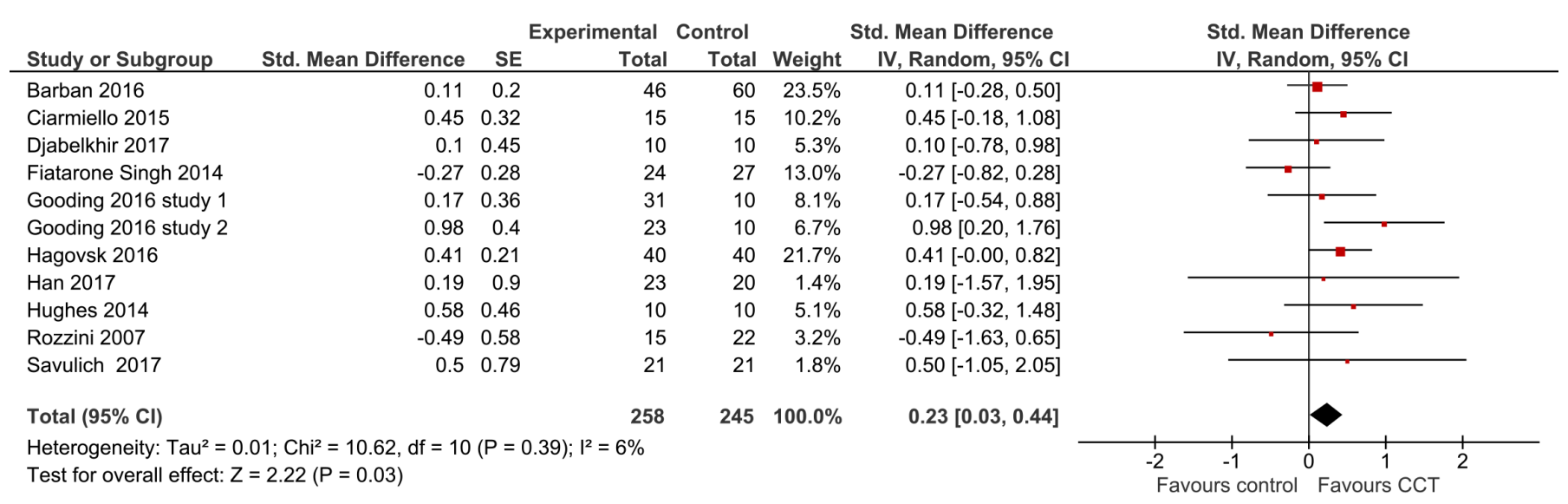

Figure 2 Forest plot demonstrating the efficacy of CCT on global cognition function. CCT, computerised cognitive training; IV, interval variable. 


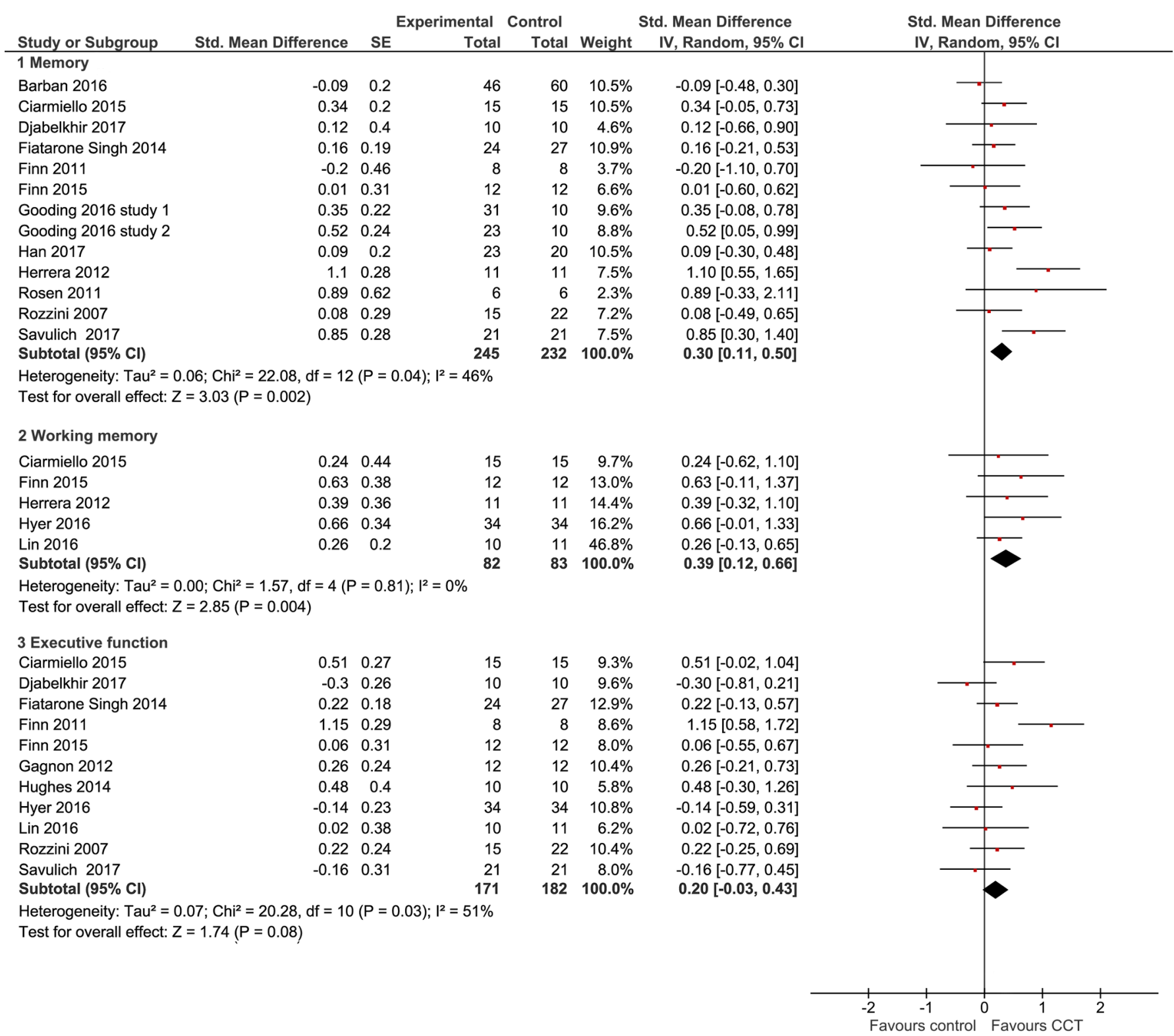

Figure 3 Forest plot demonstrating the efficacy of CCT on memory, working memory and executive function. CCT, computerised cognitive training; IV, interval variable.

( $\mathrm{n}=8, \mathrm{~g}=0.36,95 \%$ CI 0.11 to $0.61, \mathrm{I}^{2}=52 \%$ ) was larger than that of trials with passive control groups $(\mathrm{n}=5, \mathrm{~g}=0.20$, $95 \%$ CI -0.14 to $0.54, \mathrm{I}^{2}=43 \%$ ) (see online supplementary figures 6 and 7 ), but was not statistically significantly different $(\mathrm{z}=-0.32, \mathrm{p}=0.75)$. However, there was moderate heterogeneity across studies in both analyses.

Due to the moderate heterogeneity between studies, a sensitivity analysis was also conducted, in which one study at a time was removed and the others analysed to estimate whether the results could have been markedly affected by a single study. The combined Hedges' $g$ was consistent and without apparent fluctuation, with a range from 0.23 $(0.07,0.39)$ to $0.35(0.15,0.55)$.

\section{Working memory}

The meta-analysis revealed a statistically significant moderate effect size of 0.39 in favour of CCT compared with controls (95\% CI 0.12 to $0.66, \mathrm{z}=2.85, \mathrm{p}=0.004)$ with low heterogeneity between studies $\left(\mathrm{I}^{2}=0 \%\right)$ (see figure 3 ). The funnel plot did not reveal significant asymmetry (see online supplementary figure 5). Due to there being fewer than three non-active, we did not compare the effect size between active-controlled trials and non-active trials.

\section{Executive function}

The overall effect of CCT on executive function compared with control conditions was small and non-significant. The meta-analysis revealed a pooled effect size of 0.20 (95\% CI -0.03 to $0.43, \mathrm{z}=1.74, \mathrm{p}=0.08$ ) with high heterogeneity between studies $\left(I^{2}=51 \%\right.$ ) (see figure 3 ). The funnel plot did not reveal significant asymmetry (see online supplementary figure 5 ). The effect size across active-controlled trials $(\mathrm{n}=7, \mathrm{~g}=0.13,95 \% \mathrm{CI}-0.08$ to $0.35, \mathrm{I}^{2}=20 \%$ ) was smaller than for the non-active control 
groups $\left(\mathrm{n}=4, \mathrm{~g}=0.32,95 \% \mathrm{CI}-0.23\right.$ to $0.87, \mathrm{I}^{2}=74 \%$ ) (see online supplementary figures 8 and 9 ), but was not statistically significantly different $(\mathrm{z}=0.95, \mathrm{p}=0.35)$.

Considering the large heterogeneity between studies $\left(\mathrm{I}^{2}=51 \%\right)$, a sensitivity analysis was also conducted as described above. The combined Hedges' $\mathrm{g}$ was consistent and without apparent fluctuation, with a range from 0.12 $(-0.05,0.28)$ to $0.35(0.03,0.48)$.

\section{A priori subgroup analysis}

A priori, we stipulated that meta-analysis would only be performed if three studies report outcomes in the same cognitive domain and so subgroup analysis could only compare single and multidomain memory training. Similarly, only global cognition could be used for subgroup analysis to compare the training interventions less than 10 hours and more than 30 hours. Our subgroup analyses and meta-regression suggested that there is no difference between multidomain CCT and single-domain CCT ( $\mathrm{z}=0.09, \mathrm{p}=0.93$ ), although the former had a significant effect ( $\mathrm{g}=0.30,95 \% \mathrm{CI} 0.08$ to 0.53 ) while the latter was non-significant ( $\mathrm{g}=0.31,95 \% \mathrm{CI}-0.19$ to 0.81 ) (see online supplementary figures 10 and 11 ). There is also no clear evidence for a dose-response relationship. Our subgroup analysis found that studies that provided more than 30 hours of CCT had a smaller overall effect on global cognitive function $(\mathrm{g}=0.20,95 \%$ CI -0.31 to 0.71 ) compared with studies providing less than 10 hours of CCT ( $\mathrm{g}=0.30,95 \% \mathrm{CI}-0.01$ to 0.61 ) (see online supplementary figures 12 and 13). We did not perform a meta-regression for training dose because fewer than 10 studies were included. The subgroup analyses need to be interpreted with caution due to the small number of studies and heterogeneity, however, they illustrate the lack of clear factors that are associated with efficacy.

\section{DISCUSSION}

\section{Main findings}

Based on results from 18 RCTs, it is likely that CCT is a viable intervention for improving cognition in older people with MCI. There were small to moderate positive effect sizes found in all domains, with statistical significance reached for global cognitive function $(\mathrm{g}=0.23$, $95 \%$ CI 0.03 to 0.44 ), memory ( $\mathrm{g}=0.30,95 \%$ CI 0.11 to 0.50 ) and working memory ( $\mathrm{g}=0.39,95 \%$ CI 0.12 to 0.66 ), but not executive function ( $\mathrm{g}=0.20,95 \%$ CI -0.03 to $0.43)$. The largest effect sizes were found for working memory and memory (although statistically significant heterogeneity was found for the latter domain). This is unsurprising given its central focus in most interventions and promising given this is the primary complaint in most cases of MCI.

The present meta-analyses updated the literature search and added eight new studies ${ }^{23}$ 36-38 52-54 compared with the previous study conducted by Hill et $a l^{32}{ }^{32}$ The present findings are largely in keeping with the results of Hill $e t a l^{32}$ that demonstrated positive effect sizes for global cognition ( $\mathrm{g}=0.38,95 \%$ CI 0.14 to 0.62 ), memory ( $\mathrm{g}=0.42,95 \%$ CI 0.21 to 0.63 ), working memory $(\mathrm{g}=0.74$, $95 \% \mathrm{CI} 0.32$ to 1.15 ) and executive function ( $\mathrm{g}=0.20,95 \%$ CI -0.05 to 0.44 ). However, our results are in contrast with the results reported by Gates $e t a l$, which found that there were no clear effects of CCT on cognition for people with MCI. ${ }^{39}$ Methodological reasons for this inconsistency may be that Gates et al only included studies with a minimum intervention period of 12 weeks and included a broader range of participants at risk of cognitive decline. As a result, fewer studies (eight) met their eligibility criteria, of which two studies did not require a strict MCI diagnosis ${ }^{4647}$ and one used self or informant-reported cognitive complaints. ${ }^{55}$

The current meta-analysis employed strict eligibility criteria to overcome the methodological issues reported in the literature ${ }^{5657}$ such as inappropriate control groups and CCT being combined with other interventions. The combination of an overall large sample size $(n=690)$ and stringent eligibility criteria make this meta-analysis a useful contribution to the growing evidence for the efficacy of CCT in MCI. Nevertheless, various methodological issues were identified that limit the ability to make recommendations for the optimal format, frequency or intensity of CCT. Further, the lack of longitudinal studies makes it unclear whether observed postintervention benefits contribute in any way to the goal of delaying or preventing the progression from MCI to dementia.

\section{Validity of observations and limitations \\ Sources of bias}

Several methodological issues were identified. Studies were rarely double blinded, and while it may be considered impractical to blind therapists and participants given the nature of the intervention, this nevertheless introduces the risk of expectation bias and exaggerated results. In addition, data concerning drop-outs were rarely included in the analyses and intention-to-treat (ITT) analysis was only used in two studies. ${ }^{49}{ }^{58}$ While most of the remaining studies reported no significant differences at baseline for those who dropped out, these differences may have only become apparent postintervention, and baseline differences may have been more obvious with the large number of participants in the meta-analysis. Thus, the absence of ITT may have introduced an attrition bias.

Further bias may have arisen due to the decision in this study not to differentiate between amnestic and non-amnestic forms of MCI. This classification is an example of the heterogeneity of MCI symptoms. This heterogeneity is supported in descriptions by Petersen ${ }^{59}$ and in the results of a study revealing MCI as a highly nuanced and complex clinical entity. ${ }^{60}$ This may lead to considerably different intervention effects between participants and render it difficult to evaluate the efficacy of the cognitive intervention and the generalisability of the current results.

This meta-analysis calculated composite effect sizes when multiple outcome measures were provided for the 
same domain in each study. While this method maximises the amount of data drawn from the reviewed studies, it also has certain limitations. First, this approach necessitated an arbitrary measure of correlation between outcome measures, in this case, set at 0.5 . This may be inaccurate, with outcome measures being more or less heterogeneous. Unfortunately, data on composite heterogeneity were not available, however, choosing between outcome measures to decide which best represents a particular domain would have posed a significant risk of selection bias. This partly stems from the fact that 'gold-standard' tests for the different cognitive domains have not been identified.

Another limitation of the present meta-analysis is the lack of registration on PROSPERO. The registration could ensure that the protocol and results are available to other researchers for replication and updating. ${ }^{61}$ However, unfortunately at the stage of registration of our protocol, data extraction was complete and the study was, therefore, ineligible to be registered on PROSPERO.

The literature suggests multiple factors may influence the efficacy of cognitive interventions. ${ }^{62}$ An aim of the current analysis was to provide insight regarding CCT design choices and training outcomes to inform decisions on interventions to use both clinically and in future studies. Of note, the subgroups analyses and meta-regression did not find any significant differences between studies with active and non-active control conditions for any domain, or between multidomain and single-domain CCT. Due to the limited number of studies and heterogeneity of interventions and outcome measures, it is difficult to make clear recommendations for the optimal form of CCT.

This meta-analysis has demonstrated the efficacy of CCT in MCI patients for a very specific outcome: performance on a neuropsychological test immediately postintervention. While promising, this is far removed from the goal of slowing progression to or preventing dementia in MCI patients. There was a lack of follow-up data, with only three studies ${ }^{50} 5363$ including long-term outcome measures, so no conclusions can be drawn regarding the longevity of the small to moderate effects or the transfer of immediate effects. In addition, benefits on neuropsychological testing may not translate to clinically meaningful benefits in everyday function. Barnett and Ceci ${ }^{64}$ describe the immediate outcomes measured here as 'near transfer' and the long-term transfer to untrained cognitive abilities as 'far transfer'. If there is any possibility of dementia being prevented or delayed using CCT then 'far transfer' of some sort is likely necessary. A review by Zelinski ${ }^{65}$ outlines how 'far transfer' from cognitive training has been observed in ageing population, though this is not specific to CCT or MCI. Demonstration of 'far transfer' as a result of cognitive training in healthy adults is very rare and there is increasing evidence that even 'near transfer' is difficult to demonstrate convincingly. ${ }^{66}$ More research into long-term transfer effects of CCT in patients with MCI is vital in determining its potential to reduce the dementia burden.

\section{Suggestions for future research}

The discussion highlights factors limiting the reliability and transferability of the results of the meta-analysis. These limitations may be potentially overcome by more RCTs examining long-term cognitive outcomes to assess transfer of CCT to everyday life and provide more insight on whether CCT can influence progression to dementia. It is feasible to conduct large and longitudinal studies of CCT, as it can be delivered online and therefore be easily and widely available. The standardisation of outcome measures between RCTs would also avoid problems associated with heterogeneity and overall higher methodological quality of RCTs would reduce bias.

\section{CONCLUSION}

This meta-analysis has demonstrated support for the hypothesis that CCT improves cognitive function in older people with MCI. However, the long-term transfer of these improvements and relevance to reducing dementia prevalence remains unknown. Various methodological issues, such as heterogeneity in outcome measures, interventions and MCI symptoms and lack of ITT analyses, are significant limitations of the literature. Long-term outcomes are the next priority for CCT in MCI patients to further explore its efficacy with respect to influencing dementia progression.

Contributors $\mathrm{HZ}, \mathrm{RB}, \mathrm{JH}, \mathrm{RLG}, \mathrm{HW}, \mathrm{XY}$ and $\mathrm{RH}$ all contributed to the conception and design of the review. $\mathrm{HZ}, \mathrm{BH}, \mathrm{JC}$ and $\mathrm{JH}$ read and screened abstracts and titles of potentially relevant studies. $\mathrm{HZ}, \mathrm{RB}$ and $\mathrm{JH}$ read the retained papers and were responsible for extracting data and rating their quality independently. $\mathrm{HZ}$ drafted the paper with all the authors critically reviewing it and suggesting amendments prior to submission. All the authors had access to all the data in the study and can take responsibility for the integrity of the reported findings.

Funding We acknowledge the funding provided by Beijing Municipal Science and Technology Commission (no Z161100000516001, D171100008217007). HZ is supported by the China Scholarship Council (CSC) (no 201706010329) to be a visiting PhD student at University College London, UK. RB is supported by an NIHR Academic Clinical Fellowship. JH, RLG and RH are supported by the NIHR UCLH BRC.

Competing interests None declared.

Patient consent for publication Not required.

Provenance and peer review Not commissioned; externally peer reviewed.

Data sharing statement Details of excluded papers are available from the first author on request.

Open access This is an open access article distributed in accordance with the Creative Commons Attribution Non Commercial (CC BY-NC 4.0) license, which permits others to distribute, remix, adapt, build upon this work non-commercially, and license their derivative works on different terms, provided the original work is properly cited, appropriate credit is given, any changes made indicated, and the use is non-commercial. See: http://creativecommons.org/licenses/by-nc/4.0/.

\section{REFERENCES}

1. Alzheimer's Disease International. World Alzheimer Report 2015, 2015.

2. Huckans M, Hutson L, Twamley E, et al. Efficacy of cognitive rehabilitation therapies for mild cognitive impairment $(\mathrm{MCl})$ in older 
adults: working toward a theoretical model and evidence-based interventions. Neuropsychol Rev 2013;23:63-80.

3. Brookmeyer R, Johnson E, Ziegler-Graham K, et al. Forecasting the global burden of Alzheimer's disease. Alzheimers Dement 2007;3:186-91.

4. Petersen RC, Smith GE, Waring SC, et al. Mild cognitive impairment: clinical characterization and outcome. Arch Neurol 1999;56:303-8.

5. Gauthier S, Reisberg B, Zaudig M, et al. Mild cognitive impairment. Lancet 2006;367:1262-70.

6. Petersen RC. Mild Cognitive Impairment. Continuum 2016;22:404-18.

7. Karakaya T, Fußer F, Schröder J, et al. Pharmacological treatment of mild cognitive impairment as a prodromal syndrome of Alzheimer's Disease. Curr Neuropharmacol 2013;11:102-8.

8. Gates N, Valenzuela M. Cognitive exercise and its role in cognitive function in older adults. Curr Psychiatry Rep 2010;12:20-7.

9. Ball K, Berch DB, Helmers KF, et al. Effects of cognitive training interventions with older adults: a randomized controlled trial. JAMA 2002;288:2271-81.

10. Kueider AM, Parisi JM, Gross AL, et al. Computerized cognitive training with older adults: a systematic review. PLoS One 2012;7:e40588.

11. Jak AJ, Seelye AM, Jurick SM. Crosswords to computers: a critical review of popular approaches to cognitive enhancement. Neuropsychol Rev 2013;23:13-26.

12. Owen AM, Hampshire A, Grahn JA, et al. Putting brain training to the test. Nature 2010;465:775-8.

13. Hughes TF, Flatt JD, Fu B, et al. Interactive video gaming compared with health education in older adults with mild cognitive impairment: a feasibility study. Int J Geriatr Psychiatry 2014;29:890-8.

14. Gates NJ, Sachdev PS, Fiatarone Singh MA, et al. Cognitive and memory training in adults at risk of dementia: a systematic review. BMC Geriatr 2011:11:55.

15. Huntley JD, Gould RL, Liu K, et al. Do cognitive interventions improve general cognition in dementia? A meta-analysis and metaregression. BMJ Open 2015;5:e005247.

16. Wilson RS, Mendes De Leon CF, Barnes LL, et al. Participation in cognitively stimulating activities and risk of incident Alzheimer disease. JAMA 2002;287:742-8.

17. Belleville S. Cognitive training for persons with mild cognitive impairment. Int Psychogeriatr 2008;20.

18. Mowszowski L, Hermens DF, Diamond K, et al. Cognitive training enhances pre-attentive neurophysiological responses in older adults 'at risk' of dementia. J Alzheimers Dis 2014:41:1095-108.

19. Reijnders J, van Heugten C, van Boxtel M. Cognitive interventions in healthy older adults and people with mild cognitive impairment: a systematic review. Ageing Res Rev 2013;12:263-75.

20. Günther VK, Schäfer P, Holzner BJ, et al. Long-term improvements in cognitive performance through computer-assisted cognitive training: a pilot study in a residential home for older people. Aging Ment Health 2003;7:200-6.

21. Cipriani G, Bianchetti A, Trabucchi M. Outcomes of a computerbased cognitive rehabilitation program on Alzheimer's disease patients compared with those on patients affected by mild cognitive impairment. Arch Gerontol Geriatr 2006;43:327-35.

22. González-Palau F, Franco M, Bamidis P, et al. The effects of a computer-based cognitive and physical training program in a healthy and mildly cognitive impaired aging sample. Aging Ment Health 2014;18:838-46.

23. Rosen AC, Sugiura L, Kramer JH, et al. Cognitive training changes hippocampal function in mild cognitive impairment: a pilot study. $J$ Alzheimers Dis 2011:26(Suppl 3):349-57.

24. Eckroth-Bucher M, Siberski J. Preserving cognition through an integrated cognitive stimulation and training program. $A m J$ Alzheimers Dis Other Demen 2009;24:234-45.

25. Galante E, Venturini G, Fiaccadori C. Computer-based cognitive intervention for dementia: preliminary results of a randomized clinical trial. G Ital Med Lav Ergon 2007;29(3 Suppl B):B26-32.

26. Gaitán A, Garolera M, Cerulla N, et al. Efficacy of an adjunctive computer-based cognitive training program in amnestic mild cognitive impairment and Alzheimer's disease: a single-blind, randomized clinical trial. Int J Geriatr Psychiatry 2013;28:91-9.

27. Zhuang JP, Fang $R$, Feng $X$, et al. The impact of human-computer interaction-based comprehensive training on the cognitive functions of cognitive impairment elderly individuals in a nursing home. $J$ Alzheimers Dis 2013;36:245-51.

28. Bavelier D, Green CS, Han DH, et al. Brains on video games. Nat Rev Neurosci 2011;12:763-8.

29. $\mathrm{Li} \mathrm{H}, \mathrm{Li} \mathrm{J}$, Li N, et al. Cognitive intervention for persons with mild cognitive impairment: a meta-analysis. Ageing Res Rev 2011;10:285-96.
30. Martin M, Clare L, Altgassen AM, et al. Cognition-based interventions for healthy older people and people with mild cognitive impairment. Cochrane Database Syst Rev 2011;1:CD006220.

31. Simon SS, Yokomizo JE, Bottino CM. Cognitive intervention in amnestic Mild Cognitive Impairment: a systematic review. Neurosci Biobehav Rev 2012;36:1163-78.

32. Hill NT, Mowszowski L, Naismith SL, et al. Computerized cognitive training in older adults with mild cognitive impairment or dementia: a systematic review and meta-analysis. Am J Psychiatry 2017:174:329-40.

33. Sherman DS, Mauser J, Nuno M, et al. The efficacy of cognitive intervention in Mild Cognitive Impairment (MCI): a meta-analysis of outcomes on neuropsychological measures. Neuropsychol Rev 2017;27:440-84.

34. Coyle H, Traynor V, Solowij N. Computerized and virtual reality cognitive training for individuals at high risk of cognitive decline: systematic review of the literature. Am J Geriatr Psychiatry 2015;23:335-59.

35. Ge S, Zhu Z, Wu B, et al. Technology-based cognitive training and rehabilitation interventions for individuals with mild cognitive impairment: a systematic review. BMC Geriatr 2018;18:213.

36. Djabelkhir L, Wu YH, Vidal JS, et al. Computerized cognitive stimulation and engagement programs in older adults with mild cognitive impairment: comparing feasibility, acceptability, and cognitive and psychosocial effects. Clin Interv Aging 2017;12:1967-75.

37. Han JW, Son KL, Byun HJ, et al. Efficacy of the Ubiquitous Spaced Retrieval-based Memory Advancement and Rehabilitation Training (USMART) program among patients with mild cognitive impairment: a randomized controlled crossover trial. Alzheimers Res Ther 2017;9:8.

38. Savulich G, Piercy T, Fox C, et al. Cognitive training using a novel memory game on an ipad in patients with Amnestic Mild Cognitive Impairment (aMCI). Int J Neuropsychopharmacol 2017;20:624-633.

39. Gates NJ, Vernooij RW, Di Nisio M, et al. Computerised cognitive training for preventing dementia in people with mild cognitive impairment. Cochrane Database Syst Rev 2019;3:CD012279.

40. Petersen RC, Doody R, Kurz A, et al. Current concepts in mild cognitive impairment. Arch Neurol 2001;58:1985-92.

41. Higgins JPT GS, Cochrane handbook for systematic reviews of interventions version 5.1.0: The Cochrane Collaboration, 2011.

42. Hedges LV OI. Statistical methods for meta-analysis. New York: Academic Press, 1985

43. Higgins JP, Thompson SG, Deeks JJ, et al. Measuring inconsistency in meta-analyses. BMJ 2003;327:557-60.

44. Borenstein M. Introduction to meta-analysis, 2009

45. Moher D, Liberati A, Tetzlaff J, et al. Preferred reporting items for systematic reviews and meta-analyses: the PRISMA statement. BMJ 2009;339:b2535.

46. Optale G, Urgesi C, Busato V, et al. Controlling memory impairment in elderly adults using virtual reality memory training: a randomized controlled pilot study. Neurorehabil Neural Repair 2010;24:348-57.

47. Barnes DE, Yaffe K, Belfor N, et al. Computer-based cognitive training for mild cognitive impairment: results from a pilot randomized, controlled trial. Alzheimer Dis Assoc Disord 2009;23:205-10

48. Chandler MJ, Locke DEC, Duncan NL, et al. Computer versus compensatory calendar training in individuals with mild cognitive impairment: functional impact in a pilot study. Brain sciences 2017;7:10.

49. Han JW, Lee H, Hong JW, et al. Multimodal cognitive enhancement therapy for patients with mild cognitive impairment and mild dementia: a multi- center, randomized, controlled, double-blind, crossover trial. J Alzheimers Dis 2017;55:787-96.

50. Fiatarone Singh MA, Gates N, Saigal N, et al. The Study of Mental and Resistance Training (SMART) study - resistance training and/ or cognitive training in mild cognitive impairment: a randomized, double-blind, double-sham controlled trial. J Am Med Dir Assoc 2014;15:873-80.

51. Gagnon LG, Belleville S. Training of attentional control in mild cognitive impairment with executive deficits: results from a double-blind randomised controlled study. Neuropsychol Rehabil 2012;22:809-35.

52. Ciarmiello A, Gaeta MC, Benso F, et al. FDG-PET in the evaluation of brain metabolic changes induced by cognitive stimulation in $\mathrm{aMCl}$ Subjects. Curr Radiopharm 2015;8:69-75.

53. Hyer L, Scott C, Atkinson MM, et al. Cognitive Training Program to Improve Working Memory in Older Adults with $\mathrm{MCl}$. Clin Gerontol 2016;39:410-27.

54. Lin F, Heffner KL, Ren P, et al. Cognitive and neural effects of vision-based speed-of-processing training in older adults with 
amnestic mild cognitive impairment: a pilot study. J Am Geriatr Soc 2016;64:1293-8.

55. Kwok T, Wong A, Chan G, et al. Effectiveness of cognitive training for Chinese elderly in Hong Kong. Clin Interv Aging 2013;8:213-9.

56. Papp KV, Walsh SJ, Snyder PJ. Immediate and delayed effects of cognitive interventions in healthy elderly: a review of current literature and future directions. Alzheimers Dement 2009;5:50-60.

57. Martin M, Clare L, Altgassen AM, et al. Cognition-based interventions for healthy older people and people with mild cognitive impairment. Cochrane Database Syst Rev 2011;288:CD006220.

58. Vidovich MR, Lautenschlager NT, Flicker L, et al. The PACE study: a randomized clinical trial of cognitive activity strategy training for older people with mild cognitive impairment. Am J Geriatr Psychiatry 2015;23:360-72.

59. Petersen RC. Mild cognitive impairment clinical trials, 2003.

60. Libon DJ, Xie SX, Eppig J, et al. The heterogeneity of mild cognitive impairment: a neuropsychological analysis. J Int Neuropsychol Soc 2010;16:84-93.

61. Booth A, Clarke M, Dooley G, et al. The nuts and bolts of PROSPERO: an international prospective register of systematic reviews. Syst Rev 2012;1:2.

62. Solfrizzi V, Panza F, Colacicco AM, et al. Vascular risk factors, incidence of $\mathrm{MCl}$, and rates of progression to dementia. Neurology 2004;63:1882-91.

63. Herrera C, Chambon C, Michel BF, et al. Positive effects of computer-based cognitive training in adults with mild cognitive impairment. Neuropsychologia 2012;50:1871-81.
64. Barnett SM, Ceci SJ. When and where do we apply what we learn? A taxonomy for far transfer. Psychol Bull 2002;128:612-37.

65. Zelinski EM. Far transfer in cognitive training of older adults. Restor Neurol Neurosci 2009;27:455-71.

66. Stojanoski B, Lyons KM, Pearce AAA, et al. Targeted training: converging evidence against the transferable benefits of online brain training on cognitive function. Neuropsychologia 2018;117.

67. Barban F, Annicchiarico R, Pantelopoulos S, et al. Protecting cognition from aging and Alzheimer's disease: a computerized cognitive training combined with reminiscence therapy. Int $J$ Geriatr Psychiatry 2016;31:340-8.

68. Finn $\mathrm{M}, \mathrm{McD}$ onald S. Computerised cognitive training for older persons with mild cognitive impairment: a pilot study using a randomised controlled trial design. Brain Impair 2011;12:187-99.

69. Finn $\mathrm{M}, \mathrm{McD}$ conald $\mathrm{S}$. Repetition-lag training to improve recollection memory in older people with amnestic mild cognitive impairment. A randomized controlled trial. Neuropsychol Dev Cogn B Aging Neuropsychol Cogn 2015;22:244-58.

70. Gooding AL, Choi J, Fiszdon JM, et al. Comparing three methods of computerised cognitive training for older adults with subclinical cognitive decline. Neuropsychol Rehabil 2016;26(5-6):810-21.

71. Hagovská M, Olekszyová Z. Impact of the combination of cognitive and balance training on gait, fear and risk of falling and quality of life in seniors with mild cognitive impairment. Geriatr Gerontol Int 2016;16:1043-50.

72. Rozzini L, Costardi D, Chilovi BV, et al. Efficacy of cognitive rehabilitation in patients with mild cognitive impairment treated with cholinesterase inhibitors. Int J Geriatr Psychiatry 2007;22:356-60. 